www.jmscr.igmpublication.org

Index Copernicus Value: 79.54

ISSN (e)-2347-176x ISSN (p) 2455-0450

crossrefDOI: https://dx.doi.org/10.18535/jmscr/v7i1.201

\title{
A rare case of Langerhans Cell Sarcoma in a child mimicking a Lymphoma
}

\author{
Authors \\ Akkamahadevi Patil, Champaka G*, Usha A, Premalata C.S, Malathi Mukunda Pai, \\ Rekha V. Kumar, Padma Maneya \\ Department of Pathology, Kidwai Cancer Institute, Bengaluru, India \\ *Corresponding Author \\ Champaka G \\ Department of Pathology, Kidwai Cancer Institute, Bengaluru, India \\ Email: gchampaka@yahoo.co.in, Mob: 08971907498
}

Abstract
Langerhans cell sarcoma (LCS) is a high grade neoplasm with overtly malignant features of Langerhans
type cells that express S-100, CDla and Langerin and show Birbeck granules by ultrastructural
examination. They belong to the histiocytic and dendritic cell family which are antigen presenting cells and
occur in skin, bone marrow, lymph nodes, spleen, liver, and lung. While the benign form, Langerhans cell
histocytosis (LCH) is more common, the malignant form LCS is a rarity. We present a rare case of LCS in a
child with lymph node involvement.
Keywords: Langerhans cell, Malignant, Sarcoma.

\section{Introduction}

Langerhans cell sarcoma (LCS) is a rare, highly aggressive neoplasm of Langerhans cell origin. It has overtly malignant features cytologically compared to Langerhans cell histiocytosis (LCH) 1. A case of LCS of primarily nodal origin and single organ involvement is discussed here, although LCS commonly present at extranodal sites with multi-organ involvement ${ }^{(1-6)}$.

\section{Case Report}

A 11 year old girl presented with single lymph node enlargement of one year duration. There was a history of similar swelling in the past year, biopsied elsewhere for which no records were available. However there was no history of fever, weight loss or any other complaints. On clinical examination, she was a moderately built and nourished child with no pallor, icterus or edema. A single enlarged cervical lymph node at level 2 measuring $2.5 \mathrm{x} 1 \mathrm{~cm}$ was present. It was non tender, firm and mobile. The skin over the swelling was unremarkable. There were no other enlarged lymph nodes, hepatosplenomegaly, abdominal masses or skin lesions either.

\section{On Investigations}

Complete blood counts, liver function tests, renal function tests, $\mathrm{LDH}$ and random blood sugar were all within normal limits. Serology for HIV, HbsAg and $\mathrm{HCV}$ were negative. On Chest X-ray and Ultra sound abdomen no abnormality was detected. Fine needle aspiration cytology of the cervical lymph node revealed a high grade 
malignant neoplasm of probable histiocytic origin (fig1). A probable differentials of a) Histiocytic neoplasm b) Anaplastic Large Cell Lymphoma (ALCL) were considered.

Lymph node excision biopsy showed an effaced architecture with diffuse infiltration of large, pleomorphic neoplastic cells with moderate cytoplasm, vesicular nuclei, prominent nucleoli, nuclear indentations and multiple nucleoli .High mitotic activity with occasional atypical forms were noted too (fig 2,3). The morphologic differential diagnosis considered were Malignant histiocytosis, ALCL and metastatic undifferentiated carcinoma. On immunohistochemistry (IHC) the neoplastic cells were negative for epithelial markers (CK, EMA) which ruled out metastatic carcinoma, myeloid markers (CD117 ,MPO), lymphoid markers (CD79a, Pax5,CD3, CD20,CD5, Tdt) ruling out myeloid and lymphoid neoplasms, follicular dendritic markers (CD21,CD23) ruling out follicular dendritic neoplasm and (ALK-1,CD30) ruling out ALCL. They were positive for LCA, S100, CD1a, CD68 (fig 4,5,6,7) CD10 and CD43 with a high proliferative index of $70 \%$ (fig 8). Expression of CD1a, S100, CD68 and high ki67 was in favour of Langerhans cell sarcoma. However, Langerin stain was inconclusive. Although diagnostic, electron microscopy for Birbeck granules was not feasible because the tissue was fixed in formalin.

The child was treated with 6 cycles of MAID protocol (Mesna, Doxorubicin, Ifosfamide and Decarbozine) chemotherapy regime, followed by RT. She tolerated the therapy well and has been disease free since 18 months with PETCT showing no uptake.

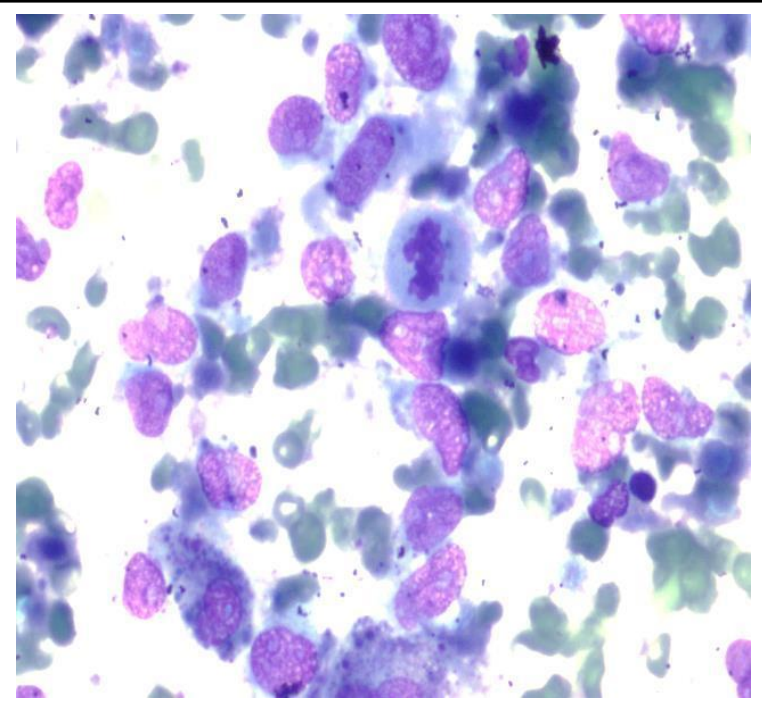

A: FNAC smear highlighting pleomorphic tumor cells with increased mitotic activity

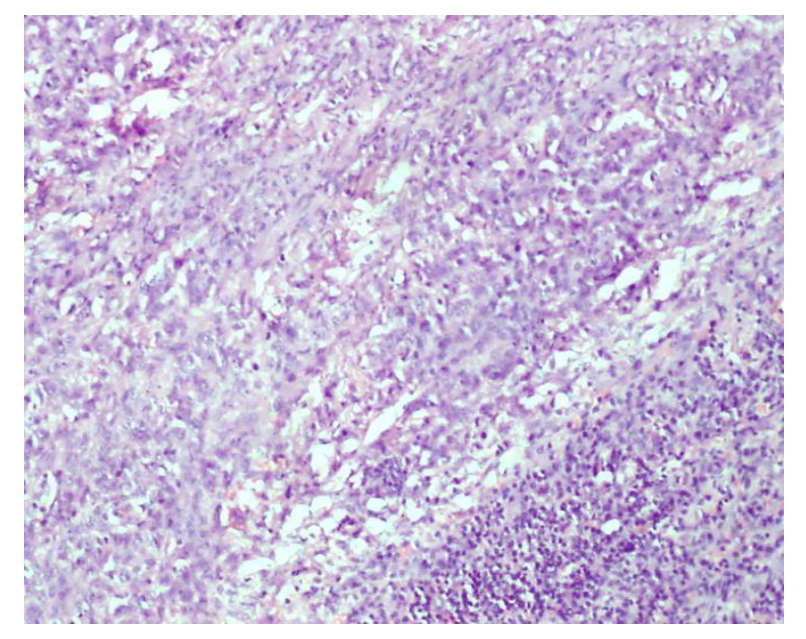

B: H\&amp; E 10X10 Lymphnode with pleomorphic tumour cells.

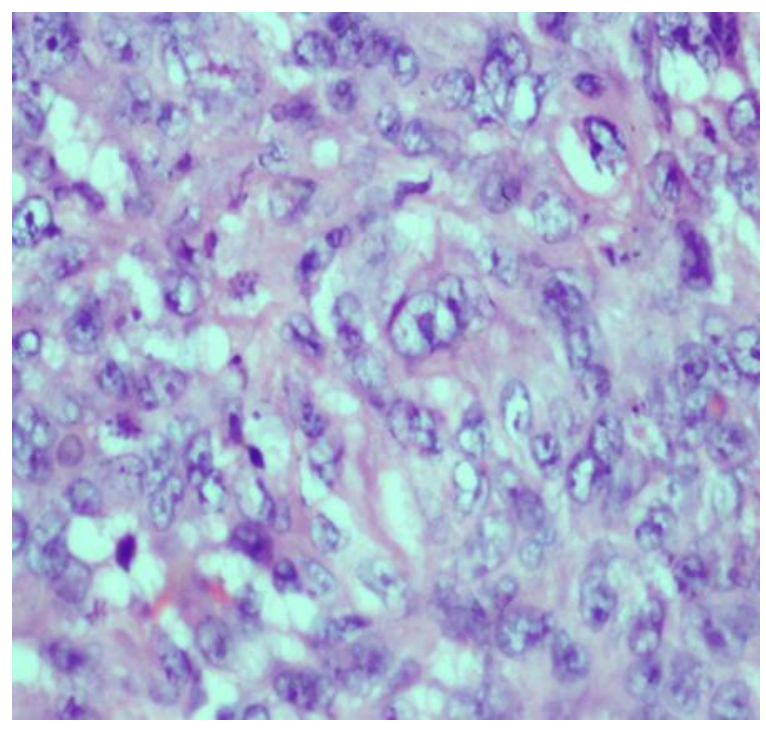

C: Biopsy, H\&amp; E,large tumor cells with indented nuclei, prominent nucleoli. H\&amp; E: $10 \times 40$. 


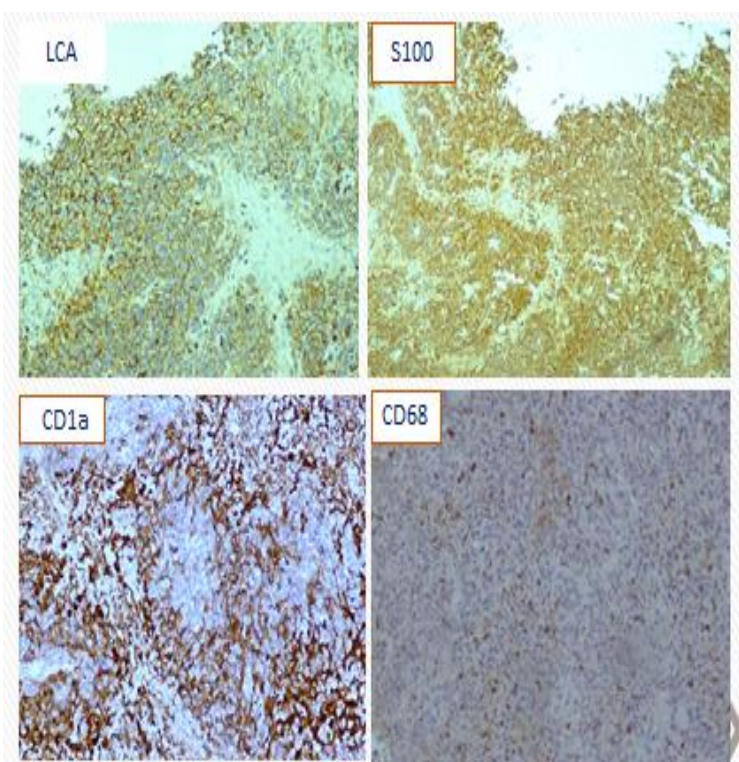

D: IHC stain : tumor cells expressing LCA ,S100, CD1a and CD68 positivity

\section{Discussion}

According to the WHO 2017 revised edition, neoplasms arising from Langerhans cell are grouped under Histiocytic/dendritic cell neoplasms ${ }^{(1)}$. Langerhans cells are a subset of hematopoietic cells thought to be derived from the monocyte-macrophage system. These are specialized dendritic cells in skin or mucosal sites devoted to antigen presention to $\mathrm{T}$ cells which upon activation migrate to lymph nodes .Lymph nodes also contain a paracortical dendritic cell type, which may be derived in part from the Langerhans cell. The classic dendritic cell lineage is believed to give rise to Langerhans cell histiocytosis/sarcoma and to interdigitating dendritic cell sarcoma ${ }^{(10)}$. Although LCH and LCS share the same phenotypic and ultrastructural features, they can be differentiated based on cytological atypia and clinical aggressiveness ${ }^{(1,2,6)}$ LCS is an overtly malignant tumour occurring in adults with the median age being 39 years, affecting males more commonly. Only 66 cases have been reported in the literature of which 46 are case series. Only a few case reports have been reported in paediatric age group. The most common sites of involvement are skin and the underlying soft tissue (extranodal). Only $22 \%$ of cases are primary nodal. Multifocal and multiorgan involvement can occur ${ }^{(7,8,9)}$.

Multifocal, high stage disease (111-1V) disease is seen in $44 \%$. In our case, it was seen in a female child, limited to a single group of cervical lymph node without cutaneous involvement or hepatosplenomegaly. Multiple sites involving bone, bone marrow, mediastinum, gall bladder, kidney, heart and thymus are reported. ${ }^{(1,2,4,6)}$

Malignant disease charecterised by cells with Langerhans cell morphology can be 1)Transition from LCH to sarcoma which occurs comonly in adults. The clinical and histopathologic onset of LCH is followed by recurrences with increasingly bizzare or anaplastic cells(which retain the CD1a phenotype)and atypical mitoses, eventuating in a frank sarcoma that may retain or loose CD1a positivity.2) Langerhans cell disease with atypical (aggressive) clinical behavior has been described after acute $\mathrm{T}$ cell Lymphoblastic leukemia and both the leukemia and the subsequent LCH showed evidence of clonality by the virtue of an identical $\mathrm{T}$ cell receptor gamma gene rearrangement. CD56 positivity has been recorded in some cases and this may help identify LCH with aggressive clinical behavior.3)Primary or denovo LC sarcoma confined almost entirely to adults, is a metastastizing tumour with poor outcome, not preceeded or accompanied by LCH .These lesions are cytologicaly malignant, but retain their Langerhans cell phenotype although staining may be less uniform than for $\mathrm{LCH}^{(1)}$ Recent studies have identified mutations in BRAF $\mathrm{V} 600 \mathrm{E}^{(12)}$ and merkel cell polyoma virus in a subset of cases. ${ }^{(11)}$

LCS is a highly aggressive tumor with an overall mean disease specific and disease free survival of 27.2(3.9) and 18.3(3.8) months respectively. In local, locoregional and disseminated disease, the disease specific and disease free survival rates vary considerably ${ }^{(5)}$. The present case was localized and did not pursue an aggressive course. Tumor progression and dissemination to extra cutaneous sites indicate an aggressive course with poor prognosis ${ }^{(5,6)}$. 


\section{Conclusion}

Although a rarity in the pediatric age group, the diagnosis of a high grade histiocytic neoplasm like LCS should also be kept in mind when a pleomorphic high grade malignant neoplasm is encountered. A wide panel of IHC markers which include histiocytic markers and EM if feasible may help in arriving at an accurate diagnosis and appropriate therapy.

\section{References}

1. Steven H. Swerdlow et al., WHO Classification of tumors of Haematopoietic and Lymphoid Tissues, 2017 Revised Edition.

2. Lakshmaiah KC, Smitha CS, Lokanatha D, Linu AJ, Premalata CS, Usha A and Aparna $\mathrm{S}$ et al. Langerhans Cell Sarcoma Review of Literature and a Rare Case Report. J Mol Biomark Diagn 2014, 5:4

3. Uchida $\mathrm{K}$ et al., Langerhans cell sarcoma emanating from the upper arm skin: successful treatment by MAID regimen. J OrthopSci 2008;13: 89-93.

4. TammieFerringer et al. Langerhans cell sarcoma. Am J Dermatopathol 2006; 28:36-39

5. Howard JEF, Dwivedi RC,Masterson L,Jani P. Langerhans cell sarcoma: A systematic review. Cancer Treat Rev (2015), 41:3320-21

6. Jong- Sil Lee et al. Langerhans Cell Sarcoma Arising from Langerhans Cell Histiocytosis: A Case Report. j Korean med sci 2006 jun: 21 (30) 577-580

7. Bohn OL,Ruiz-Arguelles G, Navarro L, Saldivar J, Sanchez-Sosa S . Cutaneous Langerhans cell sarcoma.A Case Report and review of literature. Int $J$ Hematol.2007 Feb;85(2):116-20

8. Ferringer T,Banks PM,Metcalf JS. Langerhans cell sarcoma. Am. J. Dermatopathol 2006 28(1) :36-39.
9. Pileri SA,Grogan TM, Harris NL, Banks P, Campo E, Chan JK. Tumors of histiocytic and accessory dendritic cells. An immunohistochemical approach to classification from the international lymphoma study group based on 61 cases.Histopathology 2002,41:1-29.

10. Geissmann F, Dieu-Nosjean MC, Dezutlerc, et al Accumulation of immature Langerhans cells in human lymph nodes in draining chronically inflamed skin. J. Exp.Med 2002 19;196(4):417-430.

11. Murakami I,Matsushita M,Iwasaki T,etal. High viral load of Merkel cell polyomavirus DNA sequences in Langerhans cell sarcoma issues. Infect Agent cancer 2014 9:15

12. Chen W.Jaffe R. zhang L et al( 2013) Langerhans cell sarcoma arising from Chronic lymphocytic lymphoma/Small lymphocytic leukemia. Lineage analysis and BRAF V 600E mutation study.N Am J Med Sci 2013 ;5(6):386 -391

13. Elaine Jaffe et al. Hematopathology $1^{\text {st }}$ edition ISBN: 978-0-7216-0040-6. 